Acta Crystallographica Section A

Foundations and

Advances

ISSN 2053-2733

Received 14 July 2013

Accepted 26 September 2013

\section{Experimental determination of core electron deformation in diamond}

\author{
Niels Bindzus, ${ }^{\text {a }}$ Tine Straasø, ${ }^{\mathrm{b}}$ Nanna Wahlberg, ${ }^{\text {a Jacob Becker, }}{ }^{\text {a }}$ Lasse Bjerg, ${ }^{\text {a }}$ \\ Nina Lock, ${ }^{c}$ Ann-Christin Dippel ${ }^{\mathrm{d}}$ and Bo B. Iversen ${ }^{\mathrm{a} *}$
}

\begin{abstract}
${ }^{a}$ Center for Materials Crystallography, Department of Chemistry and iNANO, Langelandsgade 140, Aarhus C 8000, Denmark, 'b Niels Bohr Institute, Universitetsparken 5, Copenhagen 2100, Denmark, 'Department of Inorganic Chemistry, Georg-August-Universität Göttingen, Tammannstrasse 4, D-37077 Göttingen, Germany, and dPETRA III, DESY Photon Science, Notkestrasse 85, D-22607 Hamburg, Germany. Correspondence e-mail: bo@chem.au.dk
\end{abstract}

\begin{abstract}
Synchrotron powder X-ray diffraction data are used to determine the core electron deformation of diamond. Core shell contraction inherently linked to covalent bond formation is observed in close correspondence with theoretical predictions. Accordingly, a precise and physically sound reconstruction of the electron density in diamond necessitates the use of an extended multipolar model, which abandons the assumption of an inert core. The present investigation is facilitated by negligible model bias in the extraction of structure factors, which is accomplished by simultaneous multipolar and Rietveld refinement accurately determining an atomic displacement parameter (ADP) of $0.00181(1) \AA^{2}$. The deconvolution of thermal motion is a critical step in experimental core electron polarization studies, and for diamond it is imperative to exploit the monatomic crystal structure by implementing Wilson plots in determination of the ADP. This empowers the electron-density analysis to precisely administer both the deconvolution of thermal motion and the employment of the extended multipolar model on an experimental basis.
\end{abstract}

\section{Introduction}

The electron-density (ED) distribution of a molecular-scale system is the most information rich observable in natural science. As shown by Hohenberg \& Kohn (1964), the ED uniquely defines the ground state of a system and application of this key theorem of quantum mechanics was the motivation for awarding the Nobel Prize in Chemistry. Indeed, the ED has a central role in chemistry as its conceptual basis can be constructed from this scalar function (Bader, 1994). Scientific rationalization of centuries of chemical observation has been based on fundamental concepts such as atoms, chemical bonds or functional groups, as well as their multitude of specific properties in different systems and under different conditions. For the chemist, the nature of a given interaction between atoms in a molecule is central for scientific understanding (Pauling, 1960), and in the discussion of chemical bonding the electrons of atoms are usually divided into core and valence electrons. Generally, it is the valence electrons that are considered to be of primary concern in chemical interactions; in fact, core electrons are often coined as 'inert'.

The ED of a molecular-scale system can be estimated experimentally by measurement of accurate X-ray diffraction data on suitable crystals. This provides access to observed structure factors, $F_{\text {obs }}$, which are the Fourier coefficients of the ED in the unit cell of a crystal (Coppens, 1997). Since direct
Fourier inversion requires measurement of structure factors to infinite resolution, and the minimal $d$-spacing available in diffraction is $\lambda / 2$, experimental $E D$ estimation is either performed by introducing a model for the unit-cell ED or by regulating the Fourier inversion such as in the maximum entropy method (Collins, 1982; Sakata \& Sato, 1990; Iversen et al., 1995). For the past 40 years, a wide range of ED models have been suggested for describing experimental structure factors (Stewart, 1976; Hirshfeld, 1977; Figgis et al., 1980) and the prevailing model is the so-called Hansen-Coppens (HC) multipolar model (Hansen \& Coppens, 1978). In this model, the ED of the crystal unit cell is considered as a sum over the densities of pseudo-atoms, each of which has a parameterized density:

$$
\begin{aligned}
\rho_{\text {atom }}(\mathbf{r})= & P_{\mathrm{c}} \rho_{\text {core }}(\mathbf{r})+P_{\mathrm{v}} \kappa_{\mathrm{v}}^{3} \rho_{\text {valence }}\left(\kappa_{\mathrm{v}} \mathrm{r}\right)+\sum_{l=0}^{l_{\max }} \kappa_{\mathrm{v}}^{\prime 3} R_{l}\left(\kappa_{\mathrm{v}}^{\prime} r\right) \\
& \times \sum_{m=0}^{l} P_{l m \pm} d_{l m \pm}(\theta, \varphi) .
\end{aligned}
$$

The HC model follows common wisdom in chemistry that the core electrons can be treated as inert and unperturbed, confining the focus of the modelling to valence electron features.

The ED of either isolated molecular systems or extended crystalline systems can also be assessed from theoretical 
calculations, for which many flavours and levels of both density and orbital-based theory exist. Theoretical calculations provide the static ED and this can only be compared with experiment if the thermal motion of the atoms is either deconvoluted from experiment or convoluted on theory. Many studies have explored detailed comparison between X-ray and theoretical EDs (Gatti et al., 1992; Volkov, Gatti et al., 2000; Schmøkel et al., 2012; Jørgensen et al., 2013) and it is widely recognized that the models used to describe the experimental ED are insufficient and therefore introduce a model bias. As an example, various studies during the past two decades have addressed the radial flexibility in the HC model (Figgis et al., 1993; Spackman et al., 1999; Volkov, Abramov et al., 2000) and currently, considerable efforts in the field are directed towards improving the description of the radial functions (Volkov \& Coppens, 2001; Koritsanszky \& Volkov, 2004). Only very recently has the implicit assumption about inert core electrons received attention. Based on $\mathrm{HC}$ multipolar modelling of theoretical structure factors, Fischer et al. (2011) established that systematic residual density features exist in the core region of the $\mathrm{C}$ atom in diamond and of the $\mathrm{Si}$ atom in crystalline silicon. However, if the $\mathrm{HC}$ multipolar model is extended (EHC) to include parameterization of the core electrons, virtually perfect fits to theoretical structure factors can then be obtained. The more flexible EHC model was able to reveal highly interesting polarization features of the core electrons, which reflect the chemical bonding environment in the diamond and silicon structures. Zhurov \& Pinkerton (2013) have also addressed the inadequacy of the HC model in describing the ED near nuclear positions. They resolved this issue by introducing a multipolar model with a double monopole in its valence description. It bears a mathematical resemblance to the EHC model of diamond; however, the physical meaning of its additional parameters is less apparent. Naturally, the question arises what the physical and chemical effects are of core electron polarization and whether such features are generally within reach for experiment in the form of accurate X-ray diffraction measurements. The study of core polarization features presents itself as a new frontier in chemical bonding studies.

In recent studies we explored the current limits of the X-ray ED method (Svendsen et al., 2010; Schmøkel et al., 2013). The vast majority of experimental ED studies are based on modelling of structure factors obtained from single-crystal X-ray diffraction (Koritsanszky \& Coppens, 2001; Coppens et al., 2005). However, in a previous study of diamond, we showed that application of the HC model is also feasible in the case of powder diffraction data (Svendsen et al., 2010). There are pros and cons for both single-crystal and powder diffraction studies and, in the latter case, background subtraction as well as peak overlap have generally been considered as insurmountable challenges to the multipolar method. Nevertheless, powder X-ray diffraction (PXRD) data feature other important advantages. They possess very limited extinction effects and, in principle, the low-order data containing most of the valence electron information are therefore better estimated. Moreover, PXRD data can be measured on a single scale, e.g. with an image-plate detector, whereas typical singlecrystal data are collected by measurements of thousands of detector frames each having slightly different experimental conditions and thus slightly different scale factors. Hence, PXRD may emerge as a promising method for experimental ED determination if the challenges of background subtraction and peak overlap are properly dealt with.

A couple of years ago, we initiated a research programme addressing exactly these features of PXRD. The best way to minimize the influence of background subtraction in the extraction of experimental structure factors is to eliminate the background intensity as much as possible. For a typical PXRD experiment, the fundamental lower limit for the background intensity corresponds to the Compton scattering from the sample and the total scattering from the container. The challenge is to avoid any kind of additional background intensity originating from scattering processes with other materials such as air. With this in mind, a new all-in-vacuum powder diffractometer was designed and commissioned. Initial measurements indeed showed a significant increase in the signal-to-noise ratio of the reflections as well as background intensity close to its fundamental limit (Straasø et al., 2013).

The complication of peak overlap can be addressed by considering the different effects contributing to peak broadening in PXRD data. They encompass particle size, particle strain/stress, beam divergence, sample size, beam size and pixel size of the detector. If crystallized micron-sized powders are used, the particle effects become insignificant. The instrumental broadening effects can be minimized if data are measured at highly collimated and extremely intense highenergy synchrotron sources such as PETRA III. For relatively simple crystal structures, it may therefore be feasible to measure X-ray scattering data of sufficient resolution to explore core polarization effects. In the present paper we report on our first attempts at measuring and analysing exceptionally high-resolution PXRD data collected using an all-in-vacuum powder diffractometer installed at an outstanding synchrotron source. As a test case, we focus yet again on diamond as thermal diffuse scattering effects are negligible due to its extreme hardness. This means that even room-temperature data are well suited for multipolar ED modelling.

Diamond is an excellent test material for investigating new methodologies, but one should not underestimate the general importance of understanding its chemical bonding in quantitative detail. Carbon is arguably the most important element in the periodic table, not just because of the huge number of organic compounds, but also because even the different polymorphs of the element itself have attracted immense attention. It is the subtleties of the $\mathrm{C}-\mathrm{C}$ chemical bonding that endow graphite, graphene, fullerene and diamond with their extraordinary material properties (Kroto et al., 1985; Iijima, 1991; Novoselov et al., 2004; Geim \& Novoselov, 2007). Providing a fundamental understanding of the chemical bonding in diamond is of significant interest for our ability to quantitatively understand and predict the properties of some of the most exciting materials ever discovered. 


\section{Methods}

\subsection{Theoretical computation}

The theoretical electronic structure was determined in the experimental geometry employing the DFT methods incorporated in the WIEN2k (W2k) software package (Blaha et al., 2008). Static structure factors, $F_{W 2 k}$, were obtained by Fourier transformation of the theoretical ED, and calculations were performed using the PBE functional (Perdew et al., 1996) on a $46 \times 46 \times 46 k$-grid with $R K_{\max }=10$. The ED within the atomic spheres was expanded to include spherical harmonics up to $L=10$.

\subsection{Data collection}

Crystalline, large-grain $(>1 \mu \mathrm{m})$ diamond powder was purchased from Nilaco and packed in a $0.2 \mathrm{~mm}$ glass capillary. In order to facilitate high-resolution PXRD, roomtemperature data were collected at the P02.1 beamline at PETRA III, Germany, using an all-in-vacuum diffractometer. The details of the diffractometer along with data-reduction procedures are described in Straasø et al. (2013). The wavelength was derived by measurements on a silicon single crystal $(a=5.4309 \AA)$ to be 0.20687 (2) $\mathrm{\text {. }}$.

\subsection{Simultaneous multipolar and Rietveld refinement}

The experimental PXRD data were treated by a delicate two-step procedure using the Jana2006 software (Petricek $e t$ al., 2006). The first step accurately extracts the observed structure factors, $F_{\text {obs }}$, from the PXRD diffractogram by simultaneous multipolar and Rietveld refinement. In the subsequent step, final multipolar refinements were performed against the extracted $F_{\text {obs }}$ employing the standard deviations estimated by Jana2006 in the weighting scheme. Separation of the multipolar refinement and the fitting of the PXRD pattern lowers the number of parameters from 15 to 6 or 8 , depending on the specific multipolar model.

In the Rietveld refinement setup, the peak shape was of the pseudo-Voigt type introducing two Gaussian parameters $(G U$, $G W)$ and one Lorentzian parameter $(L X)$. Because of proper integration of the Debye-Scherrer arcs (Straas $\varnothing$ et al., 2013), the peak-shape calculations included no asymmetry model and the peaks were cut at 30 times the full width at halfmaximum (FWHM). An essential feature of the collected PXRD data is their highly narrow, constantly well separated peaks with an FWHM varying merely from 0.04 to $0.05^{\circ}$. In consequence, the background was adequately modelled by linear interpolation between 62 manually selected points, upon which a Chebyshev polynomial consisting of four coefficients was added. The polarization correction was selected to be that of a linearly polarized beam.

In accordance with earlier findings by Svendsen et al. (2010), the multipolar refinement was performed with a radial function based on an $s p^{3}$-hybridized carbon atom. This atom is labelled as $\mathrm{Cv}$ in the SCM scattering data bank, which is constructed from wavefunctions fitted to a relativistic DiracFock solution (Su \& Coppens, 1998; Macchi \& Coppens, 2001).
The average value of the single $\zeta$ density exponent was determined on the basis of the presumed $s p^{3}$ configuration to be 3.156. Two different multipolar models are considered. The first corresponds to a standard $\mathrm{HC}$ model consisting of two radial screening parameters $\left(\kappa_{\mathrm{v}}\right.$ and $\left.\kappa_{\mathrm{v}}^{\prime}\right)$ and two independent multipole functions $(\mathrm{O} 2-, \mathrm{HO})$, which are allowed by symmetry up to fourth order. An additional hexadecapole function, $\mathrm{H4+}$, is constrained proportionally to $\mathrm{HO}$. The second model, referred to as EHC, expands upon the HC model by parameterization of the core density that enables the fitting of a core radial screening parameter, $\kappa_{\mathrm{c}}$, and a core population parameter, $P_{\mathrm{c}}$. This additional flexibility is required for a physically correct modelling of the core contraction and reduced cusp density of the carbon atom in diamond (Fischer et al., 2011). Electroneutrality is ensured by fixing the sum of the population parameters, $P_{\mathrm{v}}$ and $P_{\mathrm{c}}$. The cubic diamond structure was constructed in the $F d \overline{3} m$ (227) space group with the carbon atom located at $(0,0,0)$ and the local coordinate system oriented such that a positive sign of $\mathrm{O} 2$ - represents charge accumulation between nearest carbon atoms. Note that core deformation within the present multipolar models refers to the total deformation of the innermost electron density, not the individual changes in the atomic orbitals.

Even though the collected PXRD data display visible reflections to an impressive $\sin (\theta) / \lambda$ resolution of $2.07 \AA^{-1}$, it was necessary to exclude the highest-order region owing to software limitations (see supporting information ${ }^{\mathbf{1}}$ ). Hence, 54 reflections with a resolution of $1.70 \AA^{-1}$ were retrieved from the simultaneous multipolar and Rietveld refinement, and in the supplementary material a full listing of structure-factor values is given. The reconstruction of the theoretical and experimental charge densities was accomplished with Jana2006 and visualized by VESTA (Momma \& Izumi, 2011).

\section{Results and discussion}

The experimental charge-density analysis employed a twostep procedure separating the accurate $F_{\mathrm{obs}}$ extraction from the final multipolar refinements. This approach appreciably promotes a reliable investigation of fine features such as the core deformation in diamond. Removal of the whole pattern modelling reduces correlation and, in combination with leastsquares minimization based on structure factors rather than intensity counts, the final multipolar refinements are rendered more sensitive to the subtle information available in $F_{\text {obs }}$.

\subsection{Structure-factor extraction}

The collected PXRD data were recast into structure factors by simultaneous multipolar and Rietveld refinement incorporating the standard HC model. From a principle point of view, this is the proper course of action as the refinement model is rooted in the PXRD data and displays minimal bias towards a perturbed core density. Fischer et al. (2011) theo-

\footnotetext{
${ }^{1}$ Supporting information for this paper is available from the IUCr electronic archives (Reference: PC5033).
} 
Table 1

Parameter overview for the iterative series of simultaneous HC/Rietveld refinements.

Each refinement is characterized by weighted and non-weighted agreement factors for the pattern fit $\left(R_{\mathrm{P}}, R_{\mathrm{wP}}\right)$ and for the structural fit $\left(R_{\mathrm{F}}, R_{\mathrm{wF}}\right) . U_{\mathrm{iso}}(\mathrm{W})$ and $s(\mathrm{~W})$ are estimates derived from Wilson-plot fitting; the coefficient of determination was 0.998 for all linear least-square fits. Fixed values have a superscript of $\mathrm{F}$.

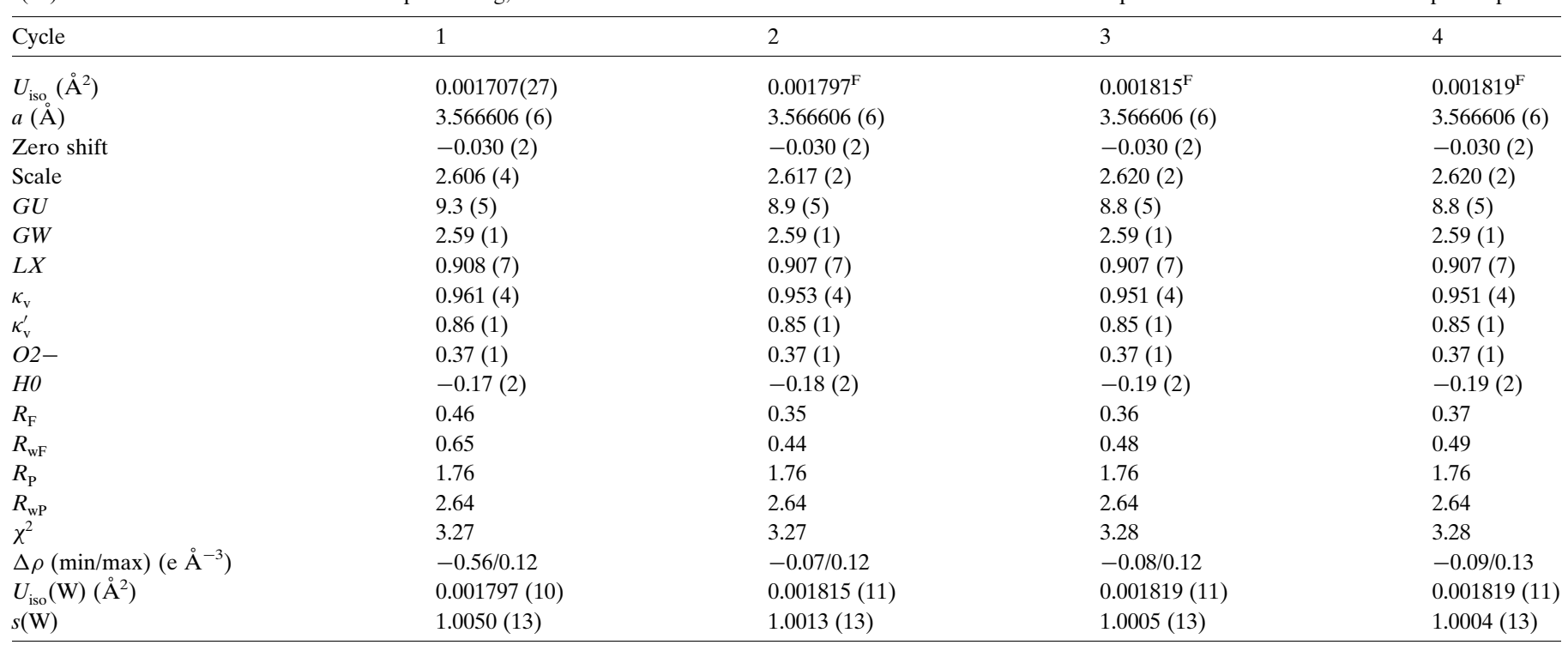

retically and experimentally established that the frozen core approximation fundamentally implemented in the HC model results in an erroneous ADP (atomic displacement parameter) that is too small. In the present case, this issue is addressed by introducing iterative comparison with theoretical static structure factors in terms of Wilson plots. Taking advantage of the monatomic diamond structure, a Wilson plot depicts the best value of the isotropic $\mathrm{ADP}, U_{\text {iso }}$, for deconvoluting the effect of thermal motion from the observed structure factors. The ADP representative of a specific set of $F_{\text {obs }}$ was obtained by applying a linear least-squares fit to the function $\ln \left(F_{\text {obs }}^{2} / F_{W 2 k}^{2}\right)=\ln (s)-16 \pi^{2} U_{\text {iso }}[\sin (\theta) / \lambda]^{2}$. In the light of a scale parameter defined so that $F_{\text {obs }} \simeq s \times F_{W 2 k}$, the intersection point evaluates the scaling introduced in the decomposition process by outlining the required correction. The possibility of deriving this additional information is exploited in $§ 3.3$, assisting considerably the exploration of core electron deformation.

The iterative procedure was initiated by retrieving the first set of $F_{\text {obs }}$ on the basis of a refinement model featuring a freely

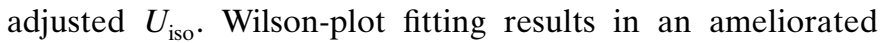
ADP, which is used at a fixed value in the subsequent refinement and $F_{\text {obs }}$ retrieval. The iterations converged quickly at a $U_{\text {iso }}$ value of $0.001819(11) \AA^{2}$ as signalled by alterations smaller than the desired accuracy of $1 \times 10^{-6} \AA^{2}$ (Table 1). The final refinement and appertaining Wilson plot analysis are depicted in Fig. 1. Interestingly, the latter displays deviations from linearity among the strong low-order reflections, which may suggest minute extinction in spite of the powder premise. At the adverse cost of peak broadening and more complicated peak overlap, extinction may be experimentally eliminated by reducing the grain sizes of the powder sample. Nevertheless, taking such action or introducing a model correction is currently unwarranted. The eminent correspondence of the final multipolar fits in $\$ 3.3$ substantiates that this potential systematic error is of negligible importance. The success of the iterative approach is corroborated by a continuously increasing ADP accompanied by substantial improvements in the structural accordance as highlighted by the agreement factors, $R_{\mathrm{F}}$ and $R_{\mathrm{wF}}$, and the residual density, $\Delta \rho(\mathbf{r})$, in Table 1. Concurring with the incapability of the $\mathrm{HC}$ model, the derived convergence point does not coincide with the closest structural fit. The agreement factors of the pattern fit, $R_{\mathrm{P}}$ and $R_{\mathrm{wP}}$, remain insensitive to the imposed ADP alterations exemplifying that fine structural features cannot fully be examined by the direct multipolar and Rietveld approach. To evaluate the HC/Rietveld extraction, a revised collection of $F_{\text {obs }}$ was retrieved by introducing the physically sounder EHC multipolar model experimentally derived in $\$ 3.3$. It displays an identical iterative behaviour converging quickly at an ADP of $0.001808(10) \AA^{2}$ (Fig. 1b) and further details are in the supplementary material. Its Wilson plot outlines minor differences, the significance of which is thoroughly analysed in the following sections.

The success of the iterative extraction process is underlined by comparison to literature reporting ADPs ranging from 0.00161 (5) to 0.002229 (4) $\AA^{2}$ (Stewart, 1973; Spackman, 1991; Yamamoto et al., 1996; Nishibori et al., 2007; Fischer et al., 2011). As clarified by both Stewart (1973) and Svendsen et al. (2010), the broad spectrum of least-squares-determined ADP values is the result of substantial correlation to the employed atomic scattering factor. The multipolar studies previously performed by Spackman (1991) and Fischer et al. (2011) led to accurate ADP determinations of 0.0018 (1) and $0.00175 \AA^{2}$, respectively. However, the former is imprecise as it is based on merely ten low-order reflections with $\operatorname{an} \sin (\theta) / \lambda$ resolution of $0.8 \AA^{-1}$, whereas the latter neglects to evaluate model bias in its structure-factor extraction. Although both 
ADP determinations concur with our results, a more appropriate reference therefore appears to be the value of 0.00189 $0.00190 \AA^{2}$ attained by lattice dynamic calculations (Stewart, 1973). Owing to thermal diffuse scattering (TDS), this value functions as an upper limit for the ADPs experimentally derived by X-ray diffraction. The large elastic constants of diamond (McSkimin \& Andreatch, 1972) delineate a small TDS correction, for which Stewart (1973) gauged a maximum value of $0.0001 \AA^{2}$. Hence, the two iteratively deduced ADPs concur with lattice dynamic calculations, establishing that model bias issues have been resolved satisfactorily.

\subsection{Structure-factor collections}

The extracted collections of observed structure factors, referred to by $F_{\mathrm{HC}}$ and $F_{\mathrm{EHC}}$, are compared to previously

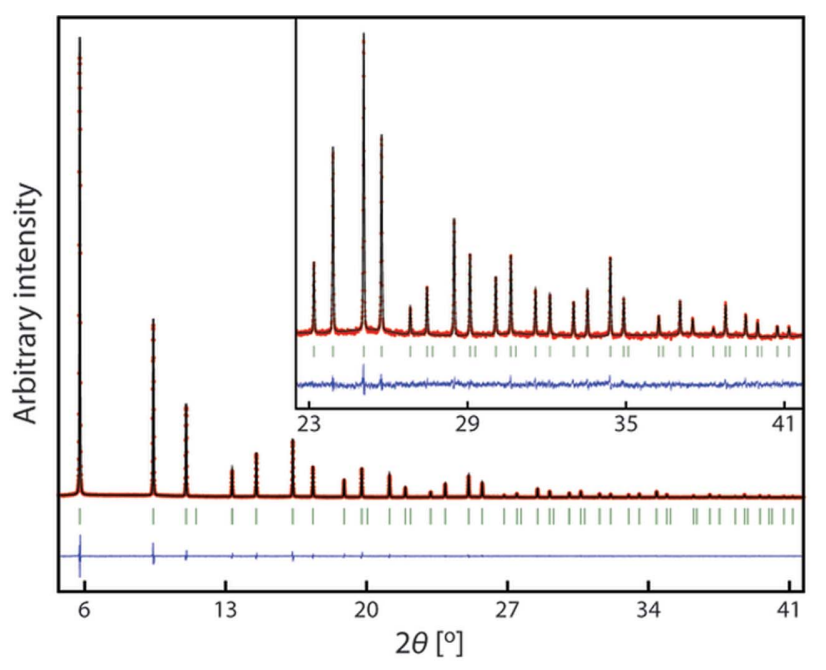

(a)

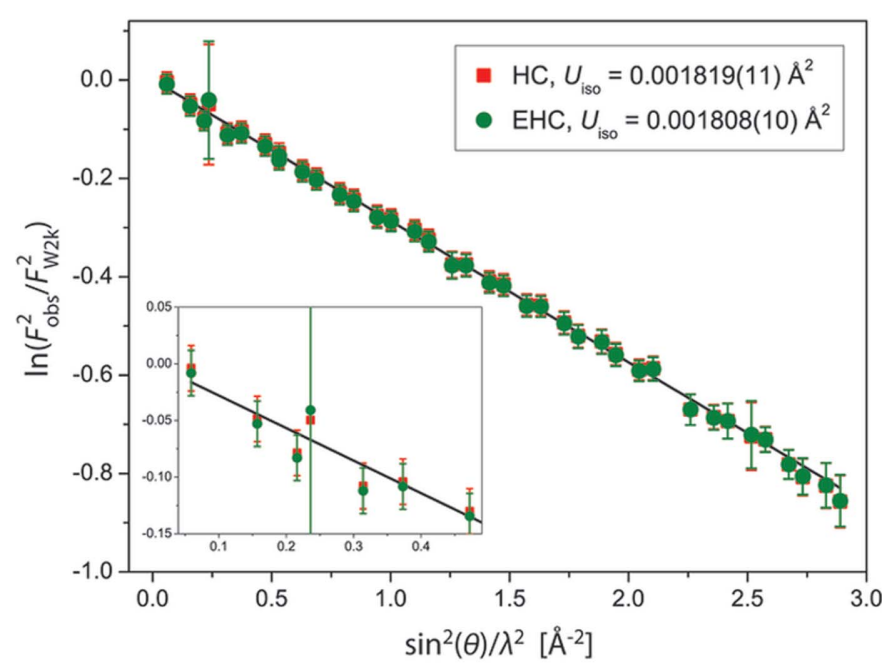

(b)

Figure 1

(a) Simultaneous $\mathrm{HC} /$ Rietveld refinement with $U_{\text {iso }}$ restricted to $0.001819 \AA^{2}$. Observed (red), calculated (black) and difference pattern (blue). (b) Wilson plots based on the $F_{\text {obs }}$ retrieved from the final $\mathrm{HC} /$ Rietveld and EHC/Rietveld extraction. The solid black line represents the linear least-square fit belonging to the HC/Rietveld case. reported high-quality data. The frame of reference comprises structure factors obtained by the Pendellösung method and PXRD at SPring8, beamline BL02B2 (Takama et al., 1990; Nishibori et al., 2007). The former data set includes nine loworder structure factors labelled as $F_{\text {Pendel }}$ and these have been analysed in detail by Spackman (1991) and Yamamoto et al. (1996). With regard to the SPring8 PXRD data, we single out the structure factors, $F_{\text {SPring } 8}$, reported by Fischer $e$ al. (2011). In line with our approach, they applied simultaneous multipolar and Rietveld refinement to decompose the PXRD pattern into 38 structure factors with a $\sin (\theta) / \lambda$ resolution of $1.45 \AA^{-1}$. The overall comparison is displayed in Fig. 2, exhibiting a reasonable agreement between $F_{\text {Pendel }}$ and $F_{\mathrm{HC}}$ without any indication of systematic deviations. With the exception of the 'forbidden' 222 reflection positioned at $0.49 \AA^{-1}, F_{\text {SPring } 8}$ concurs closely with $F_{\mathrm{HC}}$ in the region below $0.92 \AA^{-1}$ maximally deviating by $0.4 \%$. The discrepancy of the 222 reflections originates from a poorly determined $F_{\text {SPring8 }}(222)$, whose inconsistency was established relative to theoretical data (Fischer et al., 2011). However, $F_{\text {SPring8 }}$ tends

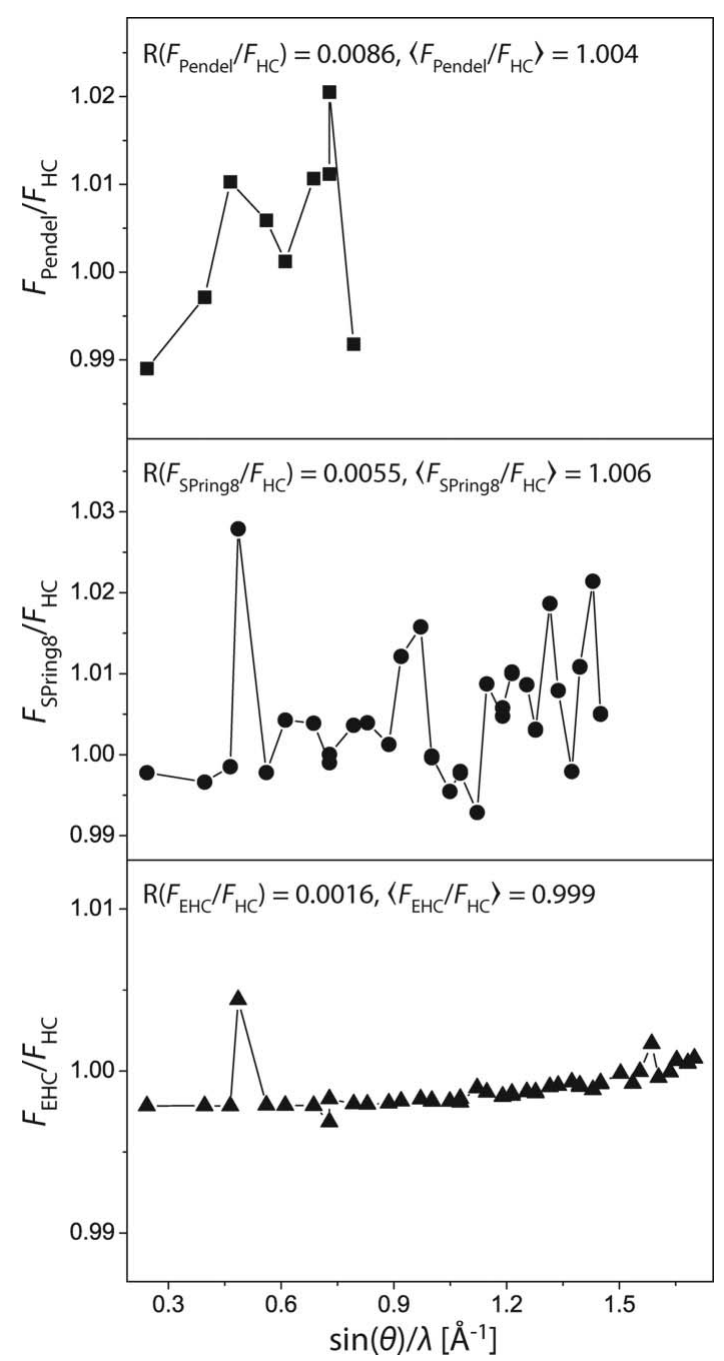

Figure 2

Comparison of different diamond data sets in terms of $F_{i} / F_{j}$ ratios, interdata-set agreement factors defined as $R\left(F_{i}, F_{j}\right)=\sum\left|F_{i}-F_{j}\right| / \sum F_{j}$ and ratio averages, $\left\langle F_{i}, F_{j}\right\rangle$. The lines act as a guide to the eye. 
Table 2

Overview of multipolar refinement performed against three different sets of structure factors: the $F_{\text {obs }}$ retrieved using (i) the HC and (ii) the EHC model in the direct multipolar/Rietveld approach and (iii) the static structure factors computed by $W 2 k$.

The superscript ${ }^{\mathrm{F}}$ denotes fixed parameters, which for the experimental case are estimated by Wilson plot fitting. Parameters evaluated at the bond critical point are denoted by the subscript ${ }_{\text {BCP. }}$.

\begin{tabular}{|c|c|c|c|c|c|c|c|c|c|}
\hline \multirow[b]{2}{*}{ Model } & \multicolumn{3}{|c|}{ HC/Rietveld extraction } & \multicolumn{3}{|c|}{ EHC/Rietveld extraction } & \multicolumn{3}{|c|}{$W 2 k$ computation } \\
\hline & $\mathrm{HC}$ & $\mathrm{HC}$ & $\mathrm{EHC}$ & $\mathrm{HC}$ & $\mathrm{HC}$ & EHC & $\mathrm{HC}$ & $\mathrm{HC}$ & EHC \\
\hline$R_{\mathrm{F}}(\%)$ & 0.37 & 0.29 & 0.26 & 0.36 & 0.28 & 0.26 & 0.23 & 0.05 & 0.03 \\
\hline$s$ & $1.0004^{\mathrm{F}}$ & $0.996(1)$ & $1.0004^{\mathrm{F}}$ & $0.9978^{\mathrm{F}}$ & $0.994(1)$ & $0.9978^{\mathrm{F}}$ & $1.0000^{\mathrm{F}}$ & 0.9951 & $1.0000^{\mathrm{F}}$ \\
\hline$\Delta \rho(\min / \max )\left(\mathrm{e} \AA^{-3}\right)$ & $-0.09 / 0.10$ & $-0.06 / 0.10$ & $-0.06 / 0.09$ & $-0.09 / 0.10$ & $-0.06 / 0.10$ & $-0.06 / 0.10$ & $-0.10 / 0.11$ & $-0.01 / 0.01$ & $-0.01 / 0.01$ \\
\hline$\kappa_{\mathrm{v}}$ & $0.952(7)$ & $0.956(7)$ & $0.958(6)$ & $0.954(7)$ & $0.956(6)$ & $0.959(6)$ & 0.961 & 0.969 & 0.971 \\
\hline HO & $-0.18(2)$ & $-0.18(2)$ & $-0.17(2)$ & $-0.18(2)$ & $-0.18(2)$ & $-0.17(2)$ & -0.123 & -0.113 & -0.105 \\
\hline$P_{\mathrm{v}}$ & $4.0^{\mathrm{F}}$ & $4.0^{\mathrm{F}}$ & $4.013(4)$ & $4.0^{\mathrm{F}}$ & $4.0^{\mathrm{F}}$ & $4.013(4)$ & $4.0^{\mathrm{F}}$ & $4.0^{\mathrm{F}}$ & 4.014 \\
\hline$\kappa_{\mathrm{c}}$ & $1.0^{\mathrm{F}}$ & $1.0^{\mathrm{F}}$ & $1.006(1)$ & $1.0^{\mathrm{F}}$ & $1.0^{\mathrm{F}}$ & $1.006(1)$ & $1.0^{\mathrm{F}}$ & $1.0^{\mathrm{F}}$ & 1.006 \\
\hline$P_{\mathrm{c}}$ & $2.0^{\mathrm{F}}$ & $2.0^{\mathrm{F}}$ & $1.987(4)$ & $2.0^{\mathrm{F}}$ & $2.0^{\mathrm{F}}$ & $1.987(4)$ & $2.0^{\mathrm{F}}$ & $2.0^{\mathrm{F}}$ & 1.986 \\
\hline$\rho_{\mathrm{BCP}}(\mathbf{r})\left(\mathrm{e} \AA^{-3}\right)$ & 1.67 & 1.67 & 1.66 & 1.67 & 1.67 & 1.66 & 1.62 & 1.61 & 1.61 \\
\hline$\nabla^{2} \rho_{\mathrm{BCP}}(\mathbf{r})\left(\mathrm{e} \AA^{-5}\right)$ & -14.05 & -13.90 & -13.61 & -14.01 & -13.86 & -13.56 & -12.15 & -11.69 & -11.41 \\
\hline
\end{tabular}

to be slightly larger in the high-order area, presumably owing to systematic errors stemming from the employment of flexible functions in its extraction process. The trend deviation manifests itself in a lower ADP for $F_{\text {SPring8, which has been }}$ derived to be $0.00175 \AA^{2}$ (Fischer et al., 2011). In the current study, the influence of model bias is minimal as verified by the high degree of similarity between $F_{\mathrm{HC}}$ and $F_{\text {EHC }}$, only deviating slightly in terms of scale and angular dependency (Fig. 2). Unsurprisingly, the 222 reflection possessing the weakest scattering intensity deviates most in the $F_{\mathrm{EHC}} / F_{\mathrm{HC}}$ trend.

\subsection{Experimental core deformation}

Multipolar refinement against the extracted $F_{\text {obs }}$ allows us to experimentally explore the core deformation phenomenon which was theoretically predicted for diamond by Fischer et al. (2011). An overview of the refinements is provided in Table 2, in which reduced structural agreement factors and residual density demonstrate significant improvements in the modelling compared with the direct multipolar and Rietveld approach.

Employing the standard HC multipolar model with both the scale and ADP constrained to the values estimated by Wilsonplot fitting, the residual density discloses an inadequate model description of the ED in the vicinity of the carbon site (Fig. $3 a$ ). Most notably, the residual density has the characteristic feature of weak positive density positioned directly at the carbon site surrounded by a large region of substantial negative density. If free adjustments of the ADP and scale parameter are allowed simultaneously, the shortcoming of the standard HC model is completely absorbed. The core features dissolve and a clearly superior description is obtained at the cost of erroneous values for $U_{\text {iso }}$ and $s$, which are reduced by 2 and $0.4 \%$, respectively (Table 2 and Fig. $3 b$ ). Introducing the
EHC model, which augments the standard HC model by parameterizing the core density, a physically correct modelling of the ED in diamond can be achieved. When the EHC model is refined subject to the $U_{\text {iso }}$ and $s$ constraint, a similar distribution of residual density and even a slightly improved structural agreement are observed compared to the unconstrained HC model (Fig. $3 c$ and Table 2). The estimated values of the core parameters, $\kappa_{\mathrm{c}}$ and $P_{\mathrm{c}}$, are 1.006 (1) and 1.987 (4), respectively, deviating slightly from their nominal values for an inert core. The EHC model necessitates restriction of the ADP and scale parameter owing to strong correlation of the order $99-100 \%$ between $s$ and $P_{\mathrm{c}}$ as well as between $\kappa_{\mathrm{c}}$ and $U_{\text {iso }}$. The constrained EHC refinement possesses only two significant correlation coefficients, namely $94.9 \%$ for $\kappa_{\mathrm{v}}^{\prime} / O 2-$ and $93.7 \%$ for $\kappa_{\mathrm{c}} / P_{\mathrm{c}}$, while the remainder are below $65 \%$.

The foundation of the experimental ED analysis is the $F_{\text {obs }}$ retrieval accomplished with the $\mathrm{HC}$ multipolar model. As decomposition of PXRD data and $I_{\text {obs }}$ to $F_{\text {obs }}$ reduction are model-dependent processes, it is essential to evaluate the impact of potential model bias. Model effects in the current analysis are gauged by conducting a revised $F_{\text {obs }}$ extraction based on the EHC multipolar model with core parameters fixed to the above experimentally determined values. As formerly established, the structural improvement introduced in the simultaneous EHC/Rietveld refinement induces minimal modifications in the extracted structure factors (Figs. $1 b$ and 2). Moreover, the similarity is retained when repeating the multipolar fits, which ultimately leads to strikingly identical observations of the core shell contraction (Table 2 and Figs. $3 d-3 f)$. The success of the HC multipolar model in retrieving highly accurate $F_{\text {obs }}$ can be understood by considering the model-dependent decomposition of overlapping reflections in greater detail. Since all peaks in the present PXRD data of diamond are well separated, it is reasonable to assume that the decomposition merely influences multiple 



\section{Figure 3}

Residual density maps, $\Delta \rho(\mathbf{r})$, in the (110) plane of the diamond lattice are shown for multipolar refinement against $(a)-(c)$ the HC/Rietveld extracted $F_{\text {obs }},(d)-(f)$ the EHC/Rietveld extracted $F_{\text {obs }}$ and $(g)-(i)$ the static structure factors. $(a),(d),(g)$ HC model subject to constraints on $U_{\text {iso }}$ and $s ;(b),(e)$, (h) flexible HC model with refined $U_{\text {iso }}$ and $s$; and $(c),(f),(i)$ EHC model subject to constraints on $U_{\text {iso }}$ and $s$. Positive (red) and negative (blue) contour

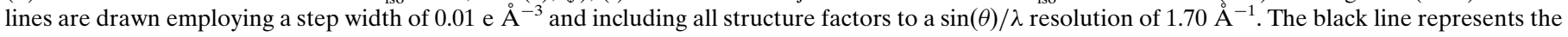
$\mathrm{C}-\mathrm{C}$ bonding chain.

peaks constructed from degenerate reflections. Therefore, for a given peak, the intensity of a reflection with reciprocallattice vector $\mathbf{H}$ will be divided from the total intensity according to a simple ratio, $r(\mathbf{H})$, expressed by

$$
r(\mathbf{H})=\frac{m_{\mathbf{H}} \cdot F_{\text {model }}^{2}(\mathbf{H})}{\sum_{\left|\mathbf{H}_{i}\right|=|\mathbf{H}|} m_{\left|\mathbf{H}_{i}\right|} \cdot F_{\text {model }}^{2}\left(\left|\mathbf{H}_{i}\right|\right)},
$$

in which $m$ refers to the multiplicity of the concerned reflections and the summation includes all reflections whose reciprocal-lattice vector has a magnitude equal to $|\mathbf{H}|$. Employing the decomposition depicted by the static structure factors as a reference, the relative performance of the $\mathrm{HC}$ and $\mathrm{EHC}$ multipolar models is displayed in Fig. 4. Their close correspondence confirms that the HC model is capable of decomposing high-order reflections containing relevant information about the core electrons as accurately as the EHC model. This feature originates from the isotropic nature of the core density in diamond. Another important reason for their similar $F_{\text {obs }}$ retrieval is the persistence of single peaks to a $\sin (\theta) / \lambda$ resolution of $1.59 \AA^{-1}$ (Fig. 4). Furthermore, the predominance of single peaks in the low-order region entails that the experimental information about the valence density, in contrast to the core density, is affected far less by model bias. In future PXRD ED studies aimed at compounds comprising elements heavier than second-row atoms, model bias is anticipated to occupy a more influential role in the $F_{\text {obs }}$ retrieval due to

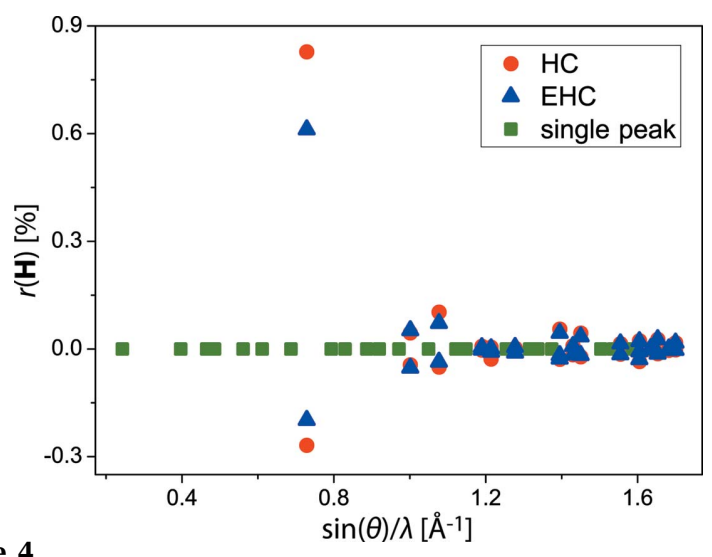

Figure 4

The deviation of the decomposition ratio $r(\mathbf{H})$ for experimentally determined $\mathrm{HC}$ and EHC multipolar models relative to the decomposition outlined by static structure factors. The positions of single peaks, containing no overlap, are shown in order to provide a complete overview of the data and to stress the scattering regions less affected by model bias. 
aspherical core deformation. For example, Fischer et al. (2011) theoretically established that silicon necessitates a multipole description up to the hexadecapole level of both the inner $M$ and $L$ shells in order to attain truly featureless residuals.

The experimental observations about core deformation are corroborated by the results of multipolar refinements performed against the static structure factors computed by $W 2 k$ (Figs. $3 g-3 i$ and Table 2). Fig. 3( $g$ ) considers the inadequacy of the standard HC model in describing the core features of diamond when $s$ and $U_{\text {iso }}$ are fixed at their true value. The residual density in the core region agrees with the experimental result, although the features are more pronounced in the theoretical map. This difference is attributable to the measurement of dynamic structure factors and counting statistics, whereas theoretical computations yield static structure factors. In the supplementary material we have deconvoluted the thermal motion from $F_{\text {obs }}$ so as to construct experimental residual maps with more distinct features. They agree even more closely with the static picture offered by theory. When $U_{\text {iso }}$ and $s$ are refined or when the standard HC model is extended to include core electrons, featureless residual density maps are obtained (Figs. 3h, 3i). The unphysical character of the HC model is revealed by its negative ADP and its scale factor deviating from unity, whereas the EHC multipolar model completely describes the core deformation of diamond using core parameters adjusted to 1.006 and 1.986 for $\kappa_{\mathrm{c}}$ and $P_{\mathrm{c}}$, respectively. Thus, theory and experiment concur excellently in the description of the subtleties in the ED of diamond. They both confirm that core-shell contraction takes place upon covalent bond formation, highlighting the inadequacy of the standard $\mathrm{HC}$ multipolar model in highresolution ED studies.

\subsection{Impact of data resolution}

It is of interest to consider the influence of data resolution when investigating core deformation in diamond, and thereby to identify the scattering region affected by errors if this phenomenon is not properly accounted for. With regard to the $\mathrm{HC}$ model, the extent of the core deformation is visible by means of its modelling failure. In the contemplation of the residual density evolution, both experimental and theoretical data show that the limit of the data resolution is a strikingly modest $0.56 \AA^{-1}$ if the $\mathrm{HC}$ model is to provide a featureless and physically correct description of the data (Fig. 5). When the data resolution is gradually increased from $0.56 \AA^{-1}$, negative features dominate the residual density until a resolution of $1.60-1.70 \AA^{-1}$, where comparable positive features emerge. This entails that even low-resolution ED studies of diamond are contaminated by systematic errors if they follow common practice and assume an inert core.

Even though the deficiency of the HC model indicates that low-resolution data may be adequate for analysing the core density, a competing factor is the number of observables. Owing to the high symmetry of diamond, a low-resolution data set comprises few structure factors and there are e.g. only 12 $F_{\text {obs }}$ below a resolution of $0.90 \AA^{-1}$. Thus, a successful EHC least-squares refinement with six adjustable parameters inevitably necessitates high-resolution data to provide a reliable determination based on experimental data containing noise. This is verified when considering the experimental derivation of $\kappa_{\mathrm{c}}$ and $P_{\mathrm{c}}$ at declining data resolution (Fig. 5). The experimental and theoretical results are in agreement starting from a resolution of $1.70 \AA^{-1}$ and down to about $1.00 \AA^{-1}$. At this point, the theoretical determination commences to diverge, whereas the experimental modelling breaks down and leaves the core deformation unsettled. The experimental EHC refinement collapses before the theoretical case attributable to experimental noise and the increasing uncertainty in the estimation of the ADP and scale parameter. As an example, the values deduced from a Wilson plot fit covering the data below $1.00 \AA^{-1}$ are $U_{\text {iso }}=0.001827(57) \AA^{2}$ and $s=0.999(3)$.

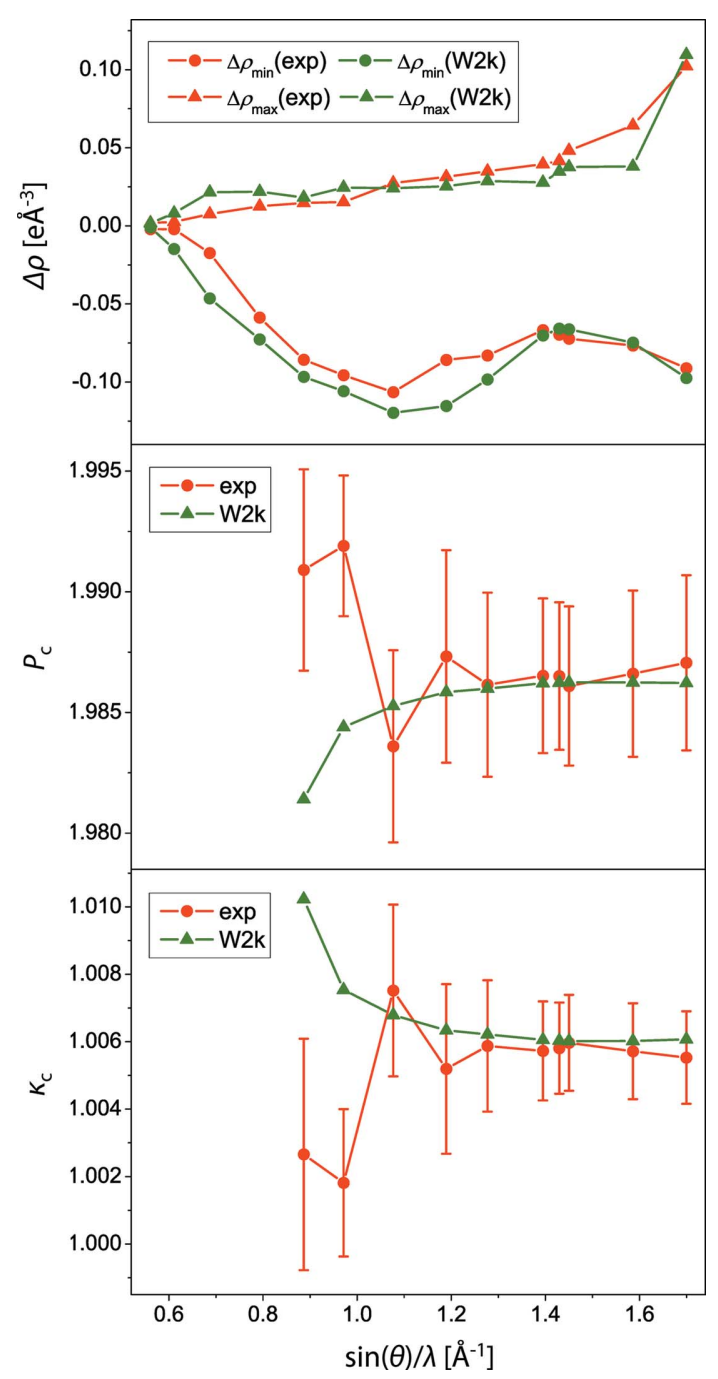

Figure 5

Variation in core deformation as a function of data resolution. The upper part depicts the maxima and minima of the residual density obtained from fitting theoretical and experimental structure factors with the $\mathrm{HC}$ model subject to constraints on $U_{\text {iso }}$ and $s$. Maintaining these restrictions and upgrading the multipolar model to EHC, the lower plots display the resulting values of the core parameters. The HC/Rietveld extracted $F_{\text {obs }}$ were selected as the experimental data. 


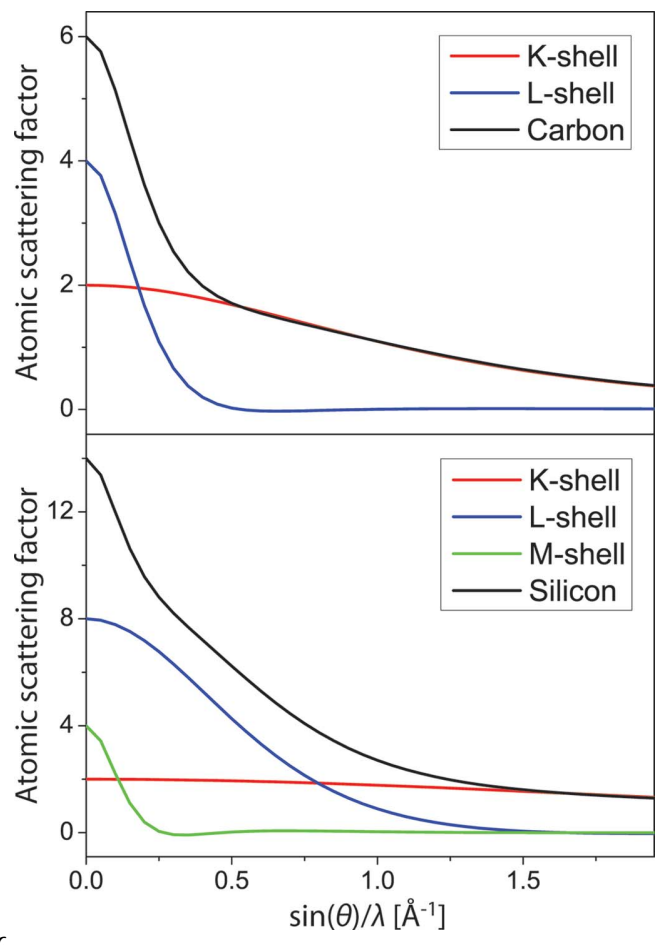

Figure 6

Scattering-factor profiles for the core and valence shell(s) in $\mathrm{C}$ and $\mathrm{Si}$ based on tabulated values computed from the SCM and VM scattering bank, respectively (Su \& Coppens, 1998; Macchi \& Coppens, 2001; Volkov et al., 2006).

The scattering curves for the valence and core electrons of diamond clarify why the influence of core deformation reaches far into the low-resolution area (Fig. 6). The scattering contribution of the core shell quickly surpasses the valence shell scattering, fading away at $c a 0.5 \AA^{-1}$. Since the first occurring diamond reflection is positioned at $0.24 \AA^{-1}$, almost all collected reflections contain a dominant core component. Contemplating more complex cases such as silicon, the requirement for the data resolution rises significantly if the scattering contributions from individual core shells are to be separated (Fig. 6). The scattering curve of silicon suggests a required data resolution above $1.50 \AA^{-1}$, and based on theoretical data it has been established that a resolution of $1.80 \AA^{-1}$ is sufficient for exploring subtle features in the shell structure of silicon (Fischer et al., 2011).

\section{Conclusion}

We report an experimental ED study pushing the limits of synchrotron PXRD, and the outstanding data quality allows us to explore the subtleties of the ED in diamond. Extending beyond a conventional description of the valence electrons, we experimentally reveal core-shell contraction inherently linked to covalent-bond formation in close correspondence with theoretical predictions. Accordingly, a precise and physically sound reconstruction of the ED in diamond necessitates the utilization of the EHC multipolar model, abandoning the assumption of an inert core. The experimental investigation is promoted by negligible model effects in the process of recasting the PXRD data into observed structure factors.
Implementation of iterative constraints in the simultaneous multipolar and Rietveld refinements results in an accurate ADP with a value of 0.00181 (1) $\AA^{2}$ in accordance with lattice dynamic calculations corrected for TDS.

The deconvolution of thermal motion is a vital step in experimental core polarization studies, especially in cases such as diamond exhibiting isotropic behaviour in both its core deformation and thermal motion. In these cases the ADP correlates strongly with the radial screening parameter of the core density, and in consequence the ADP is capable of absorbing core features. For the diamond case, simultaneous refinement of these two parameters is unstable with a correlation coefficient of $99.1 \%$ in spite of its high $\sin (\theta) / \lambda$ resolution of $1.70 \AA^{-1}$. Exploiting the monatomic crystal structure of diamond, the situation is salvaged by Wilson-plot fitting, which enables the present ED analysis to address both the deconvolution of thermal motion and the employment of the EHC multipolar model on an experimental basis.

In general, the difficulties encountered in the present study of an excellent test material outline that an experimental exploration of core polarization quickly becomes highly challenging, even for slightly more complex crystal structures. Future studies will probably be confronted by compulsory implementation of theoretically derived information in order to properly administer the more complicated deconvolution of thermal motion as well as the rapidly growing amount of subtle core parameters. The advent of high-resolution ED studies is accompanied by an increasing demand for EHC multipolar modelling. Nevertheless, our diamond study highlights that even low-resolution data contain substantial information about the core density, requiring an adequate description if systematic errors are to be prevented. In the low-order regime, the experimental precision becomes comparable to the magnitude of these errors.

We gratefully acknowledge DESY for the beam time granted at PETRA III and the Carlsberg Foundation for funding the all-in-vacuum diffractometer. The work was supported by the Danish National Research Foundation (Center for Materials Crystallography, DNRF93) and the Danish Research Council for Nature and Universe (Danscatt). Jens Als-Nielsen is thanked for fruitful discussions.

\section{References}

Bader, R. F. W. (1994). Atoms in Molecules: a Quantum Theory. Oxford University Press.

Blaha, P., Schwarz, K., Madsen, G. K. H., Kvasnicka, D. \& Luitz, J. (2008). WIEN2k, An Augmented Plane Wave + Local Orbitals Program for Calculating Crystal Properties. Technische Universität, Wien, Austria.

Collins, D. M. (1982). Nature (London), 298, 49-51.

Coppens, P. (1997). X-ray Charge Densities and Chemical Bonding. Oxford University Press.

Coppens, P., Iversen, B. \& Larsen, F. K. (2005). Coord. Chem. Rev. 249, 179-195.

Figgis, B. N., Iversen, B. B., Larson, F. K. \& Reynolds, P. A. (1993). Acta Cryst. B49, 794-806.

Figgis, B. N., Reynolds, P. A. \& Williams, G. A. (1980). J. Chem. Soc. Dalton Trans. pp. 2339-2347. 
Fischer, A., Tiana, D., Scherer, W., Batke, K., Eickerling, G., Svendsen, H., Bindzus, N. \& Iversen, B. B. (2011). J. Phys. Chem. A, 115, 13061-13071.

Gatti, C., Bianchi, R., Destro, R. \& Merati, F. (1992). J. Mol. Struct. (Theochem.), 87, 409-433.

Geim, A. K. \& Novoselov, K. S. (2007). Nat. Mater. 6, 183-191.

Hansen, N. K. \& Coppens, P. (1978). Acta Cryst. A34, 909-921.

Hirshfeld, F. L. (1977). Isr. J. Chem. 16, 226-229.

Hohenberg, P. \& Kohn, W. (1964). Phys. Rev. B, 136, 864-871.

Iijima, S. (1991). Nature (London), 354, 56-58.

Iversen, B. B., Larsen, F. K., Souhassou, M. \& Takata, M. (1995). Acta Cryst. B51, 580-591.

Jørgensen, M. R., Cenedese, S., Clausen, H. F., Overgaard, J., Chen, Y. S., Gatti, C. \& Iversen, B. B. (2013). Inorg. Chem. 52, 297-305.

Koritsanszky, T. S. \& Coppens, P. (2001). Chem. Rev. 101, 1583-1627.

Koritsanszky, T. \& Volkov, A. (2004). Chem. Phys. Lett. 385, 431434.

Kroto, H. W., Heath, J. R., O’Brien, S. C., Curl, R. F. \& Smalley, R. E. (1985). Nature (London), 318, 162-163.

Macchi, P. \& Coppens, P. (2001). Acta Cryst. A57, 656-662.

McSkimin, H. J. \& Andreatch, P. (1972). J. Appl. Phys. 43, 2944-2948.

Momma, K. \& Izumi, F. (2011). J. Appl. Cryst. 44, 1272-1276.

Nishibori, E., Sunaoshi, E., Yoshida, A., Aoyagi, S., Kato, K., Takata, M. \& Sakata, M. (2007). Acta Cryst. A63, 43-52.

Novoselov, K. S., Geim, A. K., Morozov, S. V., Jiang, D., Zhang, Y., Dubonos, S. V., Grigorieva, I. V. \& Firsov, A. A. (2004). Science, 306, 666-669.

Pauling, L. (1960). The Nature of the Chemical Bond and the Structure of Molecules and Crystals. New York: Cornell University Press.

Perdew, J. P., Burke, K. \& Ernzerhof, M. (1996). Phys. Rev. Lett. 77, 3865-3868.

Petricek, V., Dusek, M. \& Palatinus, L. (2006). Jana2006. The crystallographic computing system. Institute of Physics, Praha, Czech Republic.
Sakata, M. \& Sato, M. (1990). Acta Cryst. A46, 263-270.

Schmøkel, M. S., Bjerg, L., Overgaard, J., Krebs Larsen, F., Hellerup Madsen, G. K., Sugimoto, K., Takata, M. \& Brummerstedt Iversen, B. (2013). Angew. Chem. Int. Ed. 52, 1503-1506.

Schmøkel, M. S., Cenedese, S., Overgaard, J., Jørgensen, M. R., Chen, Y. S., Gatti, C., Stalke, D. \& Iversen, B. B. (2012). Inorg. Chem. 51, 8607-8616.

Spackman, M. A. (1991). Acta Cryst. A47, 420-427.

Spackman, M. A., Byrom, P. G., Alfredsson, M. \& Hermansson, K. (1999). Acta Cryst. A55, 30-47.

Stewart, R. F. (1973). Acta Cryst. A29, 602-605.

Stewart, R. F. (1976). Acta Cryst. A32, 565-574.

Straas $\varnothing$, T., Becker, J., Iversen, B. B. \& Als-Nielsen, J. (2013). J. Synchrotron Rad. 20, 98-104.

Su, Z. \& Coppens, P. (1998). Acta Cryst. A54, 646-652.

Svendsen, H., Overgaard, J., Busselez, R., Arnaud, B., Rabiller, P., Kurita, A., Nishibori, E., Sakata, M., Takata, M. \& Iversen, B. B. (2010). Acta Cryst. A66, 458-469.

Takama, T., Tsuchiya, K., Kobayashi, K. \& Sato, S. (1990). Acta Cryst. A46, 514-517.

Volkov, A., Abramov, Y., Coppens, P. \& Gatti, C. (2000). Acta Cryst. A56, 332-339.

Volkov, A. \& Coppens, P. (2001). Acta Cryst. A57, 395-405.

Volkov, A., Gatti, C., Abramov, Y. \& Coppens, P. (2000). Acta Cryst. A56, 252-258.

Volkov, A., Macchi, P., Farrugia, L. J., Gatti, C., Mallinson, P., Richter, T. \& Koritsanszky, T. (2006). XD2006 - a computer program for multipole refinement, topological analysis of charge densities and evaluation of intermolecular energies from experimental or theoretical structure factors.

Yamamoto, K., Takahashi, Y., Ohshima, K., Okamura, F. P. \& Yukino, K. (1996). Acta Cryst. A52, 606-613.

Zhurov, V. V. \& Pinkerton, A. A. (2013). Z. Anorg. Allg. Chem. 639, 1969-1978. 